\title{
MORFO-ANATOMÍA DE LAS HOJAS DE CELTIS EHRENBERGIANA (CELTIDACEAE) DESARROLLADAS BAJO CONDICIONES NATURALES DE SOL Y SOMBRA
}

\author{
LEANDRO NUGHES, MARTA COLARES, MARCELO HERNÁNDEZ \& ANA ARAMBARRI**
}

\begin{abstract}
Summary: Nughes, L., M. Colares, M. Hernández \& A. Arambarri. 2013. Morpho-anatomy of Celtis ehrenbergiana (Celtidaceae) leaves developed under natural light and shadow conditions. Bonplandia 22(2): 159-170.
\end{abstract}

\begin{abstract}
The aim of this paper was to establish characteristics of Celtis ehrenbergiana (Klotzsch) Liebm. (Celtidaceae) leaves developed under natural sun and shaded conditions. For the study were collected mature leaves. They were taken from the branches located in the middle of the tree crown, and inside branches between the fifth to seventh leaves from the apex of the branch. In the shade leaf, the leaf-limb is thin, herbaceous and light green. The shade leaf structure is bifacial, epidermis with wavy-sinuous anticlinal cell walls, mesophyll formed by palisade and spongy parenchyma, and angular-lacunar collenchyma. The sun leaf type has a thick leaf-limb, leathery and dark green. The sun leaf structure is equifacial, epidermis with straight anticlinal cell walls, mesophyll formed by homogeneous palisade parenchyma, and angular-massive collenchyma. There is nothing to scarcely stomata on adaxial surface in shade type leaf, however they are numerous in the sun type leaf. Celtis ehrenbergiana exhibited phenotypic plasticity. This means it has advantages over other species less adaptable and might be survive to climate changes.
\end{abstract}

Key words: Celtis ehrenbergiana, foliar epidermis, mesophyll, midrib, stomatal density.

Resumen: Nughes, L., M. Colares, M. Hernández \& A. Arambarri. 2013. Morfo-anatomía de las hojas de Celtis ehrenbergiana (Celtidaceae) desarrolladas bajo condiciones naturales de sol y sombra. Bonplandia 22(2): 159-170.

El objetivo del presente trabajo fue establecer las características de las hojas de Celtis ehrenbergiana (Klotzsch) Liebm. (Celtidaceae), desarrolladas bajo condiciones naturales de sol y de sombra. Se estudiaron hojas maduras tomadas de ramas ubicadas en la parte media de la copa del árbol y entre la quinta y séptima hoja desde el ápice. Las hojas de sombra son herbáceas, con lámina delgada de color verde claro; la estructura es bifacial, la epidermis presenta células con paredes anticlinales ondulado-sinuosas, el mesófilo está formado por parénquima en empalizada adaxial y esponjoso abaxial y el colénquima es angular-lagunar. Las hojas de sol son coriáceas, con lámina gruesa de color verde oscuro; la estructura es equifacial, la epidermis presenta células con paredes anticlinales rectas, el mesófilo es homogéneo en empalizada y el colénquima angular-masivo. En las hojas de sombra en la cara adaxial, no hay estomas o éstos son escasos, mientras que en las hojas de sol son numerosos. La variación de los caracteres morfo-anatómicos de la hoja evidenció la plasticidad fenotípica de esta especie.

'Laboratorio de Morfología Comparada de Espermatofitas (LAMCE), Facultad de Ciencias Agrarias y Forestales, Universidad Nacional de La Plata, Av. 60 y 119, C.C. 31 (1900) La Plata, Argentina.

*Autor para correspondencia: Tel. +54-221-423-6758, int. 461; Fax: +54-221-425-2346. E-mail: anaramba@yahoo. com.ar; botgral@agro.unlp.edu.ar 


\begin{abstract}
Esto indicaría las ventajas que tendría frente a otras especies menos adaptables y podría sobrevivir ante los cambios climáticos.
\end{abstract}

Palabras clave: Celtis ehrenbergiana, epidermis foliar, mesófilo, vena media, densidad estomática.

\section{Introducción}

El género Celtis L. pertenece a la familia Celtidaceae y en la Argentina está representado por cuatro especies (Zuloaga \& al., 2008). Celtis ehrenbergiana (Klotzsch) Liebm. "tala" (Berg \& Dahlberg, 2001), es un arbusto o arbolito de 3-10 (-20) $\mathrm{m}$ alt. con el tronco tortuoso, la corteza delgada con fisuras longitudinales y ramas espinosas. Las hojas son simples, brevemente pecioladas y con filotaxis alterna. La lámina es aovado-asimétrica, de 2,5-6,0 cm long. x 0,9-2,5 cm lat., con el margen entero, aserrado o dentado en la mitad distal. En el envés de la lámina son notables los domacios en el ángulo de las venas secundarias con la vena principal. La venación es broquidódroma y en ambas superficies el indumento está formado por tricomas glandulares y eglandulares con cistolito basal. Las inflorescencias son cimosas de flores verdoso-amarillentas. El fruto (pireno) es globoso anaranjado (Dottori, 1976; Demaio \& al., 2002). Es una especie de amplia distribución en la Argentina, encontrándose en las provincias de Jujuy, Salta, Santiago del Estero, Mendoza, San Juan, San Luis, Catamarca, La Rioja, Chaco, Formosa, Misiones, Corrientes, Entre Ríos, Córdoba, Santa Fe, La Pampa y Buenos Aires (Zuloaga \& al., 2008). En la provincia de Buenos Aires, C. ehrenbergiana tiene hábito gregario y ribereño. Se localiza lo largo de la costa sobre diferentes tipos de suelos, prosperando mejor sobre los calcáreos. En esta región, el área de distribución del tala está reducida por el avance de la actividad antrópica y por las dificultades para la germinación de sus semillas y crecimiento de sus plántulas. Goya \& al. (1992) y Arturi \& Goya (2004) han realizado diversos estudios con la finalidad de proteger la especie. Por otro lado, otros autores han descripto diferentes aspectos de los talares bonaerenses (Mérida \& Athor, 2006; Athor, 2009). Otras contribuciones se refieren a ensayos de micropropagación in vitro (Abedini \& al., 2000; Adema \& al., 2007) y sobre tecnología y durabilidad de la madera (Spavento \& al., 2006; Murace \& al., 2007).

La hoja es el órgano de la planta más sensible a las condiciones ambientales, por lo cual refleja alteraciones morfológicas como una forma de adaptación a los efectos del estrés (Toral $\&$ al., 2010). Los estomas se forman al inicio del crecimiento de la hoja y maduran cuando se alcanza un 10-60\% del tamaño foliar final (Royer, 2001). La iniciación de los estomas es influida por la concentración de $\mathrm{CO}_{2}$ en la atmósfera y sobre la expansión celular actúan numerosas variables como la luz, la temperatura, la posición de la hoja en la planta. Por lo tanto, la densidad estomática es afectada por el número de estomas, por el tamaño de las células epidérmicas y por la iniciación de los estomas y la expansión de las células epidérmicas (Royer, 2001). De esta manera, la densidad de estomas depende de la ubicación de la hoja dentro de la copa del árbol, el estado de desarrollo de la hoja y la parte de la lámina que se considere. Además, los factores ambientales tales como la intensidad de luz, disponibilidad de agua, humedad relativa, altitud sobre el nivel del mar, nutrientes, concentración de dióxido de carbono atmosférico inciden sobre la densidad estomática (Salisbury, 1927; Stace, 1965; Gay \& Hurd, 1975; Woodward, 1987; Bravo \& Grau, 1992; Poole \& al., 1996; Royer, 2001; Ma \& al., 2004; Hernández \& Arambarri, 2010; Arambarri \& al., 2011). De acuerdo con Fahn \& Cuttler (1992) uno de los factores principales que afectan la distribución y densidad estomática es la intensidad de radiación solar y/o intensidad de luz. Como la densidad estomática determina el área potencial de intercambio gaseoso, su variabilidad es una respuesta de considerable importancia ecofisiológica (Woodward, 1987). Dada la importancia que para la región bonaerense tienen los montes de "tala" existentes, así como la necesidad de su conservación como proyección futura, resulta de interés estudiar la plasticidad fenotípica de la especie. Los estudios 
morfo-anatómicos contribuirían a interpretar la variabilidad de respuesta de esta especie a las condiciones ambientales imperantes en diferentes localidades. Arambarri \& al. (2011) hallaron variabilidad en los caracteres foliares de plantas de $C$. ehrenbergiana que crecían en diferentes ambientes. Sobre la base de estos estudios previos se plantea como objetivo establecer las características epidérmicas y estructurales de las hojas de $C$. ehrenbergiana desarrolladas bajo condiciones naturales de sol y de sombra.

\section{Material y Métodos}

\section{Material estudiado}

Entre los días 21 y 24 de uno o dos meses correspondientes a cada estación climática del año 2010, se recolectaron hojas maduras en completo estado de desarrollo de plantas de Celtis ehrenbergiana. Las hojas fueron tomadas de las ramas ubicadas en la parte media de la copa del árbol y dentro de la rama entre la quinta y séptima hoja desde el ápice. Las plantas elegidas se encuentran situadas a pleno sol y en condiciones de plena sombra en el Jardín Botánico y Arboretum "C. Spegazzini" de la Facultad de Ciencias Agrarias y Forestales, Universidad

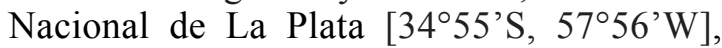
en zona templado-húmeda. Las condiciones climáticas bajo las cuales se desarrolló el estudio están registradas en la Tabla 1. Estos datos fueron provistos por el Departamento de Sismología e Información Meteorológica de la Facultad de Ciencias Astronómicas y Geofísicas, Universidad Nacional de La Plata.

Tabla 1. Registros climáticos estacionales para el año 2010: valores promedios de Temperatura $\left({ }^{\circ} \mathrm{C}\right)$, Humedad (\%), Presión atmosférica en H. Pascal (Hpa), Velocidad media del viento $(\mathrm{km} / \mathrm{h})$ y precipitación acuosa en $(\mathrm{mm})$ del área de estudio.

\begin{tabular}{lccccc}
\hline \multicolumn{1}{c}{ MES } & $\begin{array}{c}\text { Temp. } \\
{ }^{\circ} \mathrm{C}\end{array}$ & $\begin{array}{c}\text { Hum. } \\
(\%)\end{array}$ & $\begin{array}{c}\text { Pres. } \\
(\mathrm{Hpa})\end{array}$ & $\begin{array}{c}\text { Vel. } \\
(\mathrm{km} / \mathrm{h})\end{array}$ & $\begin{array}{c}\text { Lluvia } \\
(\mathrm{mm})\end{array}$ \\
\hline Verano (V) & 22,3 & 82,0 & 1010,1 & 12,0 & 164,3 \\
Otoño (O) & 14,2 & 80,3 & 1020,3 & 10,2 & 75,9 \\
Invierno (I) & 10,9 & 78,6 & 1020,8 & 12,9 & 83,2 \\
Primavera (P) & 19,1 & 73,5 & 1014,4 & 12,5 & 42,5 \\
\hline
\end{tabular}

\section{Microscopía óptica}

Las hojas frescas se fijaron en FAA (formol, agua destilada, ácido acético y alcohol etílico 2:7:1:10). Para el estudio de la epidermis en vista superficial, las hojas fueron diafanizadas a temperatura ambiente. Para ello, el material fue sumergido en una solución formada por una mezcla de hidróxido de sodio al $5 \%$ e hipoclorito de sodio al $5 \%$ en partes iguales, durante 4-5(-15) días. Posteriormente, el material fue lavado con agua destilada (AD) y decolorado con hipoclorito de sodio al 50\% hasta quedar transparente. Luego, se realizaron tres lavados con AD y fue colocado en hidrato de cloral al $5 \%$ por $24 \mathrm{~h}$ hasta su completa clarificación. Los materiales diafanizados fueron coloreados con solución alcohólica de safranina $80 \%$. Los cortes transversales fueron realizados en la parte media de la lámina. Las secciones elegidas fueron decoloradas con hipoclorito de sodio al 50\%, lavadas con agua hasta eliminar las burbujas de aire y posteriormente, coloreadas con violeta de cresilo al 1\%. Los materiales diafanizados y las secciones transversales coloreadas fueron montadas con gelatina-glicerina (Johansen, 1940; D’Ambrogio de Argüeso, 1986). Los datos de las hojas de sol como de sombra se obtuvieron sobre ocho campos ópticos ubicados en la parte media de ambos semilimbos de la lámina y sobre ambas epidermis, adaxial y abaxial. Los valores fueron expresados como promedios de 160 repeticiones por lámina foliar. Los parámetros analizados de cada epidermis en vista superficial fueron las características de las paredes anticlinales y dimensiones de las células epidérmicas (excluyendo aquellas adyacentes a los estomas y las que rodean la base de los tricomas), los tipos y distribución de los estomas, la longitud y latitud de los estomas y latitud de las células oclusivas. Los tamaños se expresan en micrómetros $(\mu \mathrm{m})$. Para establecer la densidad estomática se realizaron los recuentos de estomas, no considerando aquellos que se encontraban en el borde del campo visual quedando visible en una proporción inferior a su mitad. En el recuento de estomas de la epidermis adaxial, se incluyeron aquellos ubicados sobre las venas y próximos a ellas. La densidad de estomas 
se expresa por milímetro cuadrado (estomas/ $\mathrm{mm}^{2}$ ). En la vista superficial de la epidermis adaxial se determinó el índice de empalizada (IE). Para ello fueron contadas las células en empalizada que se encontraban debajo de cuatro células epidérmicas y se sumaron las que se hallaban en el borde cuando más de la mitad se encontraba dentro del área. Se calculó el promedio de células en empalizada ubicadas debajo de una célula epidérmica, dividiendo la sumatoria de las células en empalizada por cuatro, constituyendo el índice de empalizada (IE). Para este parámetro se realizaron 10 determinaciones por preparación y se calculó el IE promedio. En el corte transversal se establecieron las características cualitativas de la vena media y lámina foliar, tales como la prominencia de la vena media y el desarrollo de colénquima. Sobre las alas de la lámina foliar se determinó el tamaño de las células epidérmicas y el tipo y espesor del mesófilo. Para medir las dimensiones de las células epidérmicas, estomas y mesófilo se empleó un microscopio Leitz SM lux equipado con Moticam 2300 y software Motic Image Plus 2.0. Las imágenes fueron obtenidas con un microscopio Gemalux equipado con una cámara PAL CCD y digitalizadas con el software Hyper Media Center. Los resultados de los recuentos y mediciones se indican para las cuatro estaciones del año en las Tablas 2-5. Los caracteres destacables se muestran en las Figuras 1-5. La terminología usada en las descripciones morfológicas está de acuerdo con Metcalfe \& Chalk (1979).

\section{Análisis estadístico}

Sobre los datos promedio de densidad estomática total, producto de la suma de las densidades promedio de las caras adaxial y abaxial tanto de hojas de sol como de sombra, de importancia fisiológica (Parkhurst, 1978), se aplicó el análisis de la varianza (ANOVA). Asimismo, se realizó la prueba de Tukey de comparación múltiple de las medias para obtener grados de significancia del 5\% $(\mathrm{P}<0,05)$ (Tabla 4; Fig. 3). Los análisis estadísticos propuestos fueron realizados con la ayuda del software Statistica 7.0 para Windows.

\section{Resultados y Discusión}

\section{Morfología foliar}

Las hojas de sol se presentan gruesas y coriáceas, de color verde oscuro y frecuentemente incurvadas a lo largo de la vena media. Las hojas de sombra son delgadas, herbáceas, verde claro y extendidas. Los rasgos generales de la hoja (pecíolo, forma y bordes de la lámina, venación, pubescencia, presencia de cistolitos y domacios) fueron coincidentes con descripciones previas (Dottori, 1976; Fernández Honaine \& al., 2005).

\section{Anatomía foliar}

Epidermis en vista superficial. Las células epidérmicas de las hojas de sol, tanto en la cara adaxial como abaxial, presentan las paredes anticlinales rectas a levemente curvadas, mientras que en las hojas de sombra son onduladosinuosas (Fig. 1A-D). Las características de las paredes de las células epidérmicas en las hojas de sol coinciden con las reportadas por Dottori (1976), mientras que, las paredes onduladas en las células epidérmicas de las hojas de sombra concuerdan con lo citado por Stace (1965), Metcalfe \& Chalk (1979) y Roth (1984). Estos autores señalaron que los pliegues de las paredes anticlinales responden a las condiciones de luz y humedad del ambiente. Así, en las hojas que se desarrollan a la sombra estas paredes resultan sinuosas, más aún, en lugares húmedos.

Las hojas de sol presentan valores promedio de longitud y latitud de las células epidérmicas de $32,7 \times 22,6 \mu \mathrm{m}$ para la epidermis adaxial y de 22,3 x 14,1 $\mu \mathrm{m}$ para la abaxial. Mientras que en las hojas de sombra estos valores fueron de $38,1 \times 25,4 \mu \mathrm{m}$ para la epidermis adaxial y 31,9 x 19,2 $\mu \mathrm{m}$ para la abaxial. Los valores promedios muestran que en las hojas de sol, ambas epidermis tienen células epidérmicas con menor tamaño que en las hojas de sombra. Sin embargo, estas diferencias son menos notables cuando consideramos los valores de tamaño de las células epidérmicas en las estaciones climáticas, con un resultado inverso en el período estival, cuando se produjeron valores de temperatura, humedad y lluvia más elevadas (Tabla 2). En general, los estomas son anomocíticos, raramente 


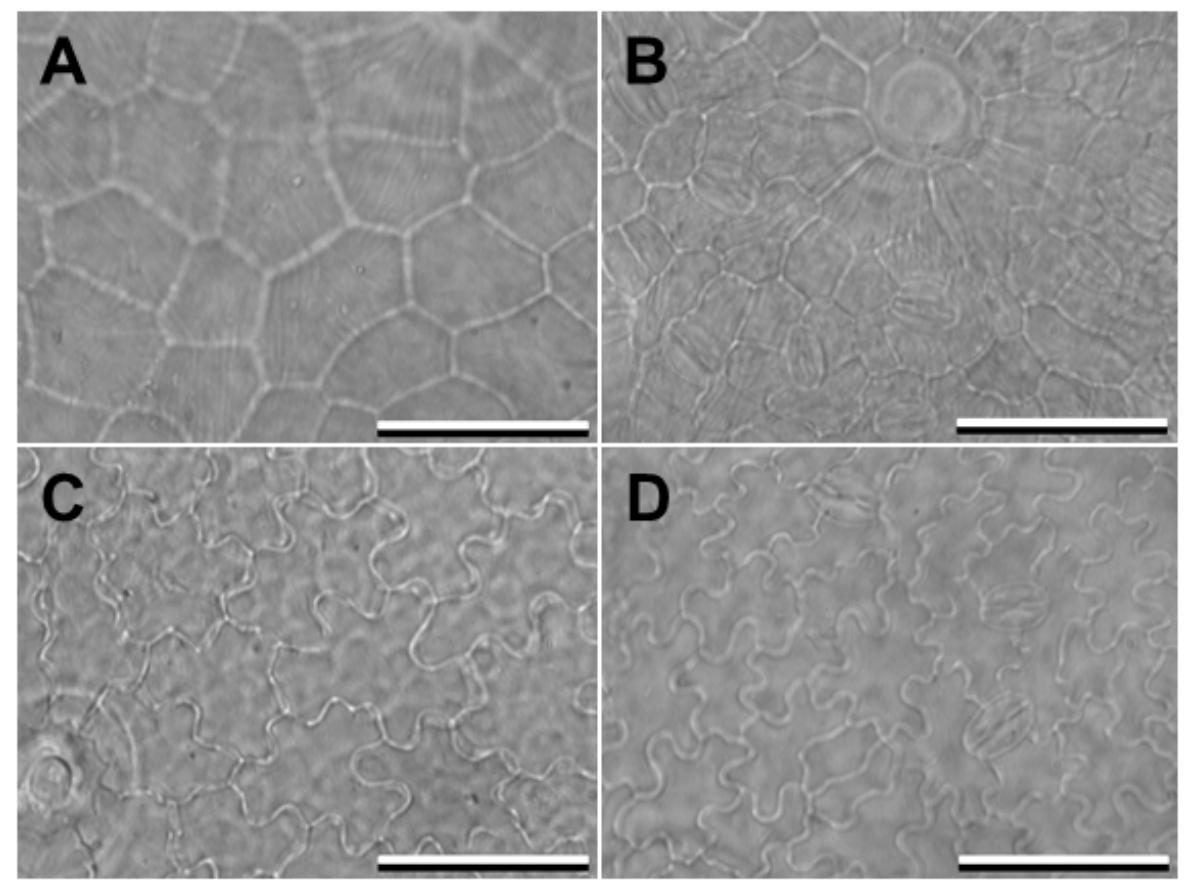

Fig. 1. Epidermis foliar en vista superficial de Celtis ehrenbergiana. Hojas de sol: A y B: Epidermis adaxial y abaxial, respectivamente; en ambas las paredes anticlinales son rectas a levemente curvadas. Hojas de sombra: C y D: Epidermis adaxial y abaxial, respectivamente; en ambas las paredes anticlinales son sinuosas. Escalas: $50 \mu \mathrm{m}$.

anisocíticos y están ubicados a nivel con respecto a las restantes células epidérmicas (Fig. 2A, B). Esta característica coincide con Dottori (1976) para C. ehrenbergiana. Las hojas de sombra no tienen estomas en la cara adaxial o éstos son escasos y se ubican sobre y en la proximidad de las venas secundarias y terciarias, mientras que las hojas de sol presentan estomas numerosos en la cara adaxial y se ubican en los espacios intervenosos. Dottori (1976) describió las hojas de $C$. ehrenbergiana como anfistomáticas.

Los valores promedio de longitud y latitud de los estomas en las hojas de sol son de 23,1 x 14,2 $\mu \mathrm{m}$ en la cara adaxial y de 18,8 x 11,9 $\mu \mathrm{m}$ en la abaxial. En las hojas de sombra los estomas presentan un tamaño de 19,6 x 12,0 $\mu \mathrm{m}$ en la cara adaxial y de $17,8 \times 11,4 \mu \mathrm{m}$ en la abaxial. Por lo tanto, los estomas de las hojas de sol son más grandes que los de las hojas de sombra. Sin embargo, si consideramos los valores promedio de longitud y latitud de los estomas en las estaciones climáticas (Tabla 2), durante el período invernal el tamaño de los estomas en las hojas de sol y de sombra son similares; en este período se produjo una reducción de la temperatura pero con valor de lluvia elevado. Por otra parte, en las hojas de sol recolectadas en primavera se hallaron estomas proporcionalmente grandes rodeados por células epidérmicas radiadas cubiertas por notables estrías cuticulares (Fig. 2B). Estos estomas podrían comportarse como hidatodos, teniendo como función secretar o absorber agua (Roth, 1984). La aparición de este tipo de estomas es coincidente con el registro de una reducción de las lluvias y humedad ambiental acompañada de un incremento de la temperatura (Tabla 1). De este modo coincide con la referencia de Roth (1984) sobre que la función de los hidátodos en un ambiente húmedo surge cuando se produce una reducción de la disponibilidad de agua.

La densidad estomática en la cara adaxial de las hojas de sol presentó un valor promedio de 174,5 estomas $/ \mathrm{mm}^{2}$, oscilando entre un mínimo de 15,1 estomas $/ \mathrm{mm}^{2}$ y un máximo de 363,6 estomas $/ \mathrm{mm}^{2}$. En la cara abaxial se determinó un valor promedio de 790,9 estomas $/ \mathrm{mm}^{2}$, con valores mínimo y máximo de 454,5 y 1075,7 estomas $/ \mathrm{mm}^{2}$ respectivamente. En las hojas de sombra la densidad estomática mostró un 
Tabla 2. Epidermis foliar de Celtis ehrenbergiana en vista superficial: dimensiones promedio de las células epidérmicas, estomas y células oclusivas en micrómetros $(\mu \mathrm{m})$ y valores del índice en empalizada (IE) para las hojas de sol y de sombra en los períodos estacionales de verano: V; otoño: O; invierno: I y primavera: P. Ref.: abx: abaxial; adx: adaxial; cel: células; epid: epidermis.

\begin{tabular}{|c|c|c|c|c|c|c|c|}
\hline \multirow{2}{*}{$\begin{array}{l}\begin{array}{l}\text { Estación/ } \\
\text { epidermis }\end{array} \\
\text { condición }\end{array}$} & \multicolumn{2}{|c|}{$\begin{array}{c}\text { Cél epidérmicas } \\
(\mu \mathrm{m})\end{array}$} & \multicolumn{2}{|c|}{$\begin{array}{c}\text { Estomas } \\
(\mu \mathrm{m})\end{array}$} & \multicolumn{2}{|c|}{$\begin{array}{c}\text { Cél oclusivas } \\
(\mu \mathrm{m})\end{array}$} & \multirow[t]{2}{*}{ IE } \\
\hline & long. & lat. & long. & lat. & lat. & lat. & \\
\hline $\mathbf{V}$ adx sol & 26,5 & 20,6 & 20,6 & 13,3 & 5,7 & 5,5 & 15 \\
\hline abx sol & 20,7 & 13,7 & 17,3 & 11,4 & 4,6 & 4,5 & \\
\hline adx sombra & 24,2 & 12,8 & 17,7 & 11,0 & 4,6 & 4,6 & 8 \\
\hline abx sombra & 26,8 & 15,7 & 15,9 & 11,4 & 4,9 & 5,0 & \\
\hline O adx sol & 36,1 & 23,5 & 25,7 & 14,4 & 6,6 & 6,7 & 14 \\
\hline abx sol & 22,9 & 14,6 & 19,1 & 12,5 & 5,5 & 5,4 & \\
\hline adx sombra & 48,4 & 34,4 & 19,3 & 10,9 & 4,5 & 4,5 & 7 \\
\hline abx sombra & 35,0 & 20,5 & 17,0 & 10,4 & 4,5 & 4,6 & \\
\hline I adx sol & 37,9 & 24,8 & 22,7 & 13,3 & 5,7 & 6,4 & 13 \\
\hline abx sol & 25,0 & 15.0 & 18,5 & 11,2 & 5,0 & 5,1 & \\
\hline adx sombra & 43,0 & 31,0 & 22,2 & 13,7 & 6,4 & 5,9 & 8 \\
\hline abx sombra & 33,3 & 19,9 & 19,7 & 11,5 & 4,9 & 5,2 & \\
\hline $\mathbf{P}$ adx sol & 30,5 & 21,6 & 23,5 & 15,8 & 6,9 & 6,7 & 14 \\
\hline abx sol & 20,7 & 13,0 & 20,5 & 12,7 & 5,8 & 5,7 & \\
\hline adx sombra & 36,8 & 23,3 & 19,4 & 12,4 & 5,4 & 5,6 & 6 \\
\hline abx sombra & 32,4 & 20,5 & 18,7 & 12,2 & 5,3 & 5,3 & \\
\hline
\end{tabular}
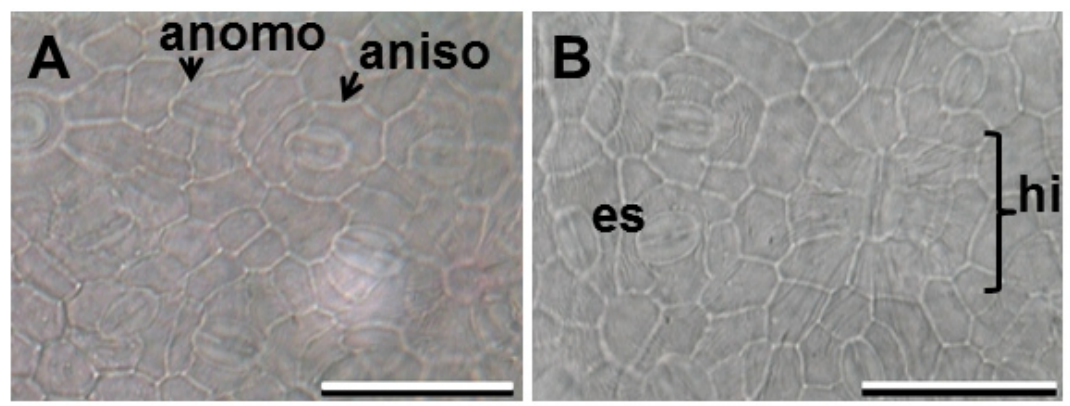

Fig. 2. Epidermis foliar en vista superficial de Celtis ehrenbergiana. A: Estoma anisocítico y estomas anomocíticos. B: Estomas anomocíticos y un hidatodo. Abreviaturas: aniso = anisocítico; anomo = anomocítico; es = estomas; hi = hidatodo. Escalas: $50 \mu \mathrm{m}$.

valor promedio de 8,3 estomas $/ \mathrm{mm}^{2}$, oscilando entre 0 y 60,6 estomas $/ \mathrm{mm}^{2}$, mientras que en la cara abaxial el valor promedio fue de 431,0 estomas $/ \mathrm{mm}^{2}$ con un mínimo y máximo de 242,4 y 651,5 estomas $/ \mathrm{mm}^{2}$. En la Tabla 3, se muestran los valores de densidad estomática promedios para cada estación. Las diferencias en densidad estomática son notables al comparar las caras adaxiales de las hojas de sol y de sombra en cualquiera de las estaciones climáticas, resultando evidente el aumento del número de estomas en la superficie adaxial de las hojas 
de sol. Por otra parte, también se observa una importante diferencia en la densidad estomática al comparar las caras abaxiales de hojas de sol y de sombra. Por último, en las hojas de sombra la diferencia de densidad estomática entre la cara adaxial y abaxial es conspicua (Tabla 3 ).

De los datos registrados se desprende que en la cara adaxial de las hojas de sol hay un aumento del número y tamaño de estomas con respecto a las hojas de sombra (Tablas 2 y 3). Los resultados coinciden con Fahn \& Cutler (1992) quienes refieren que la aparición de estomas adaxiales se produce por la intensa radiación solar. En cuanto al tamaño de los estomas (Roth, 1984, 1990) señaló que el déficit hídrico provoca reducción del tamaño de los estomas, por lo tanto, el aumento de las dimensiones de los mismos en la cara adaxial, se atribuye a la disponibilidad hídrica existente en el ambiente templado-húmedo en que se realizó el ensayo (Tabla 1). Por otra parte, al realizar la sumatoria de los valores de densidad estomática de las caras adaxial y abaxial en hojas de sol (densidad estomática total), siempre fue más elevada que la sumatoria en las hojas de sombra (Tabla 4; Fig. 3). Esto también se atribuye a las condiciones húmedas en que se desarrolló el estudio, ya que Fahn \& Cutler (1992) en ambiente xérico encontraron que los valores de densidad estomática en las hojas de sol y de sombra eran similares cuando se sumaban ambas epidermis, e igual resultado obtuvieron Arambarri \& al. (2011) al estudiar C. ehrenbergiana creciendo en la región semiárida chaqueña. Por último, la importancia de estos resultados acuerda con Parkhurst (1978) quien sugirió que la formación de estomas en la cara adaxial sumados a los de la cara abaxial incrementan la conductancia de $\mathrm{CO}_{2}$ en la hoja, por lo tanto, la alta densidad estomática hallada en hojas de sol respecto a las hojas de sombra indica que las primeras tendrían mayor eficiencia fotosintética.

Índice de empalizada (IE). El valor promedio de IE en hojas de sol es de 14, mientras que para las hojas de sombra es 7. Resultó notablemente más elevado el IE en las hojas de sol que de sombra (Tabla 2; Fig. 4A). Esto significa que hay más células en empalizada por célula epidérmica en hojas de sol y se corresponde con un mesófilo que presenta células en empalizada más largas y estrechas en el primer estrato subepidérmico (Fig.
Tabla 3. Epidermis foliar de Celtis ehrenbergiana: valores promedio de densidad de estomas por milímetro cuadrado (estomas $/ \mathrm{mm}^{2}$ ) en los períodos estacionales de verano: V; otoño: O; invierno: I y primavera: P. Epid adx: epidermis adaxial; Epid abx: epidermis abaxial.

\begin{tabular}{ccc}
\hline Hojas de sol & $\begin{array}{c}\text { Epid adx } \\
\left(\text { estomas } / \mathrm{mm}^{2}\right)\end{array}$ & $\begin{array}{c}\text { Epid abx } \\
\left(\text { estomas } / \mathrm{mm}^{2}\right)\end{array}$ \\
\hline V & 162,1 & 904,4 \\
O & 176,9 & 604,5 \\
I & 113,6 & 795,5 \\
P & 245,4 & 859,1 \\
\hline \multirow{2}{*}{ Hojas de sombra } & Epid adx & Epid abx \\
& $\left(\right.$ estomas $\left./ \mathrm{mm}^{2}\right)$ & $\left(\right.$ estomas $\left./ \mathrm{mm}^{2}\right)$ \\
\hline V & 10,6 & 563,6 \\
O & 1,5 & 487,9 \\
I & 3,0 & 287,9 \\
P & 16,7 & 384,8 \\
\hline
\end{tabular}

Tabla 4. Epidermis foliar de Celtis ehrenbergiana en vista superficial: densidad estomática total (estomas/ $\mathrm{mm}^{2}$ ) en hojas de sol y sombra, sumados los valores promedios de las epidermis adaxial y abaxial para los períodos estacionales de verano: $\mathrm{V}$; otoño: $\mathrm{O}$; invierno: I y primavera: $P$.

\begin{tabular}{lcc}
\hline & Hoja de sol & Hoja de sombra \\
\hline V & 1066,5 & 574,2 \\
$\mathbf{O}$ & 781,4 & 489,4 \\
$\mathbf{I}$ & 909,1 & 290,9 \\
$\mathbf{P}$ & 1104,5 & 401,5 \\
\hline
\end{tabular}

4B). Este carácter se halla en hojas desarrolladas bajo alta radiación solar (Roth, 1984).

Estructura foliar en corte transversal. Ambas epidermis son unistratas, están formadas por células rectangulares con la pared periclinal externa engrosada y cubierta por una cutícula estriada, tal como fue mencionado por Dottori (1976). En las hojas de sol, el tamaño promedio de altura y latitud de las células epidérmicas es de $23,4 \times 24,0 \mu \mathrm{m}$ en la cara adaxial y de $13,1 \times 15,8 \mu \mathrm{m}$ en la cara abaxial; en cambio, en las hojas de sombra es de 14,0 x 19,3 $\mu \mathrm{m}$ en la cara adaxial y de $10,7 \times 13,9 \mu \mathrm{m}$ en la cara abaxial. Los resultados muestran que las 


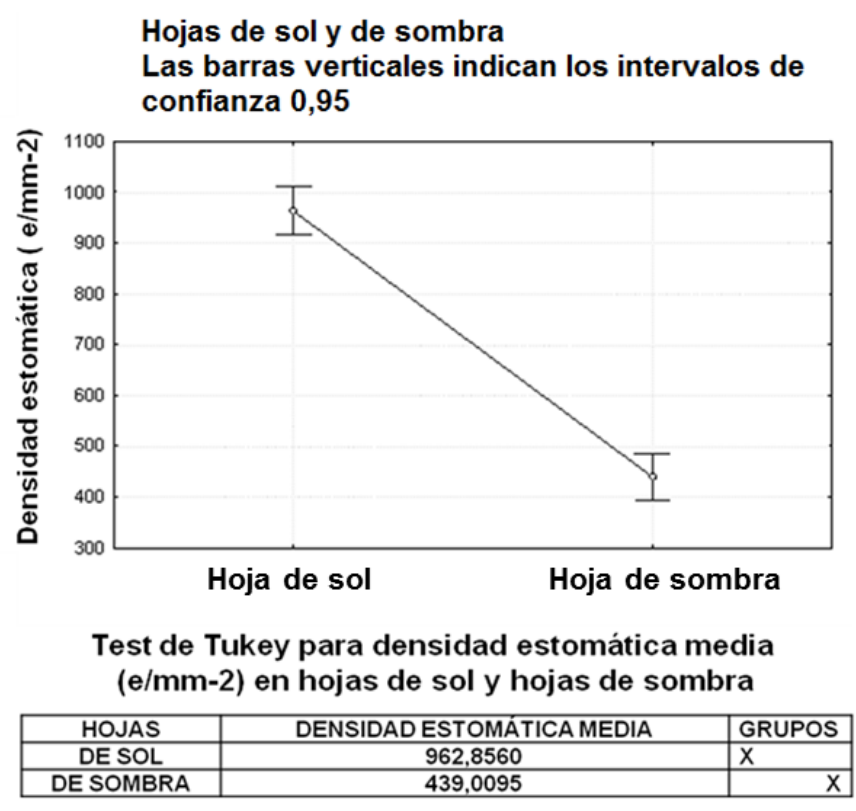

Fig. 3. Densidad media de estomas por milímetro cuadrado (estomas $/ \mathrm{mm}^{2}$ ). Análisis estadístico de los datos dados en la Tabla 4 que representa la diferencia significativa entre los valores medios obtenidos para la densidad total de estomas en las hojas de sol y de sombra.

células de la epidermis adaxial son mayores que las de la abaxial y que en las hojas de sombra las células epidérmicas de ambas epidermis tienen menor altura que latitud, mientras que en la cara adaxial de las hojas de sol las células epidérmicas tienden a igualar altura y latitud o presentan un leve alargamiento anticlinal (Tabla 5). El alargamiento anticlinal de las células epidérmicas es una adaptación xeromórfica, que se produce para contribuir a la conservación de agua y evitar la acción negativa de la excesiva insolación sobre los estratos internos de células en empalizada (Roth, 1984).

El mesófilo de las hojas de sol está formado por parénquima homogéneo en empalizada. Se observa el primer estrato adaxial de células muy largas (Fig. 4B) y en los 3-5 estratos siguientes las células también son alargadas en sentido perpendicular a la superficie, reduciendo paulatinamente su longitud hasta alcanzar la cara abaxial. El mesófilo en las hojas de sombra es dorsiventral. Se observan 1-2 estratos adaxiales de células en empalizada cortas (Fig. 4C) y en los 2-3 estratos siguientes las células son redondeadas y dejan pequeños espacios intercelulares formando un parénquima esponjoso denso. El espesor del mesófilo en las hojas de sol dio un valor promedio de 141,4 $\mu \mathrm{m}$, con valores mínimo y máximo de 116,0 y 175,2 $\mu \mathrm{m}$ y en las hojas de sombra el valor promedio fue de $56,6 \mu \mathrm{m}$ variando entre 39,3 y $72,5 \mu \mathrm{m}$ (Tabla 5). El mayor espesor del mesófilo en hojas de sol se debe principalmente a un alargamiento de las células en empalizada y no a un incremento del número de capas celulares (Fig. 4B, C). El aumento del espesor foliar, el pasaje hacia un mesófilo homogéneo en empalizada y el gran alargamiento sufrido por el primer estrato de células en empalizada son adaptaciones xeromórficas. Los resultados coinciden con lo referido por Luna \& De la Sota (2003) sobre los cambios estructurales producidos por el ambiente en la hoja de Jodina rhombifolia. Lo mismo hallaron Colares \& Arambarri (2008) al estudiar la anatomía foliar de Ziziphus mistol. Se conoce que, los caracteres xeromórficos aparecen tanto en ambiente semiárido como en ambiente húmedo, pero en este último cuando las hojas están expuestas a una alta radiación solar (Roth, 1984; Arambarri \& al., 2011). La mayor longitud de las células en empalizada contribuye a la difusión de la excesiva insolación protegiendo los estratos más internos que, al no recibir tanta radiación solar, reducen su 
longitud hacia la cara abaxial menos expuesta (Roth, 1984). Esau (1982) señaló que las plantas terrestres evolucionaron hacia la tolerancia y uso de niveles más elevados de incidencia de luz solar y la estructura foliar evolucionó hacia las llamadas hojas de sol. Asimismo, Silva \& Silva (2001) también indicaron que el clorénquima suele cambiar la forma y dimensiones de sus células como adaptación al ambiente donde la especie crece. Smith \& al. (1997) mencionaron que la máxima capacidad de fotosíntesis requiere de una eficiente distribución del $\mathrm{CO}_{2}$ y luz dentro de la hoja y que algunas de las estructuras que elevan la eficiencia para la fotosíntesis son capas de células en empalizada y estomas en la cara adaxial. De esta manera, las hojas de sol son más eficientes fotosintéticamente que las de sombra (Cosa \& Dottori, 2010). Bianco $\&$ al. (2004) también refieren que el mayor espesor del mesófilo se corresponde con una mayor concentración de clorofila, un aumento de la tasa de asimilación de $\mathrm{CO}_{2}$ y eficiencia en el uso del agua.

La vena media es biconvexa. El tejido
Tabla 5. Lámina foliar de Celtis ehrenbergiana en corte transversal: dimensiones promedio de las células epidérmicas en las caras adaxial y abaxial y espesor del mesofilo de hojas de sol y de sombra para los períodos

estacionales de verano: V; otoño: O; invierno: I y primavera: P. Abreviaturas: Epid adx: epidermis adaxial; Epid abx: epidermis abaxial.

\begin{tabular}{|c|c|c|c|c|c|c|}
\hline & \multicolumn{2}{|c|}{$\begin{array}{c}\text { Epid adx } \\
(\mu \mathrm{m})\end{array}$} & \multicolumn{2}{|c|}{$\begin{array}{c}\text { Epid abx } \\
(\mu \mathrm{m})\end{array}$} & \multirow{2}{*}{$\begin{array}{l}\text { Mesófilo } \\
\quad(\mu \mathrm{m}) \\
\text { espesor }\end{array}$} \\
\hline & & altura & latitud & altura & latitud & \\
\hline \multirow{2}{*}{ V } & & 23,5 & 27,8 & 12,5 & 15,7 & 119,6 \\
\hline & sombra & 14,9 & 22,6 & 11,4 & 14,3 & 61,0 \\
\hline \multirow{2}{*}{$\mathbf{O}$} & sol & 27,5 & 25,2 & 14,6 & 15,7 & 154,7 \\
\hline & sombra & 17,8 & 26,0 & 12,1 & 16,7 & 72,5 \\
\hline \multirow{2}{*}{ I } & sol & 19,3 & 20,4 & 14,3 & 17,3 & 175,2 \\
\hline & sombra & 11,0 & 14,6 & 07,2 & 12,1 & 39,3 \\
\hline \multirow{2}{*}{$\mathbf{P}$} & sol & 23,5 & 22,5 & 11,0 & 14,7 & 116,0 \\
\hline & sombra & 12,4 & 14,1 & 12,1 & 12,4 & 53,5 \\
\hline
\end{tabular}
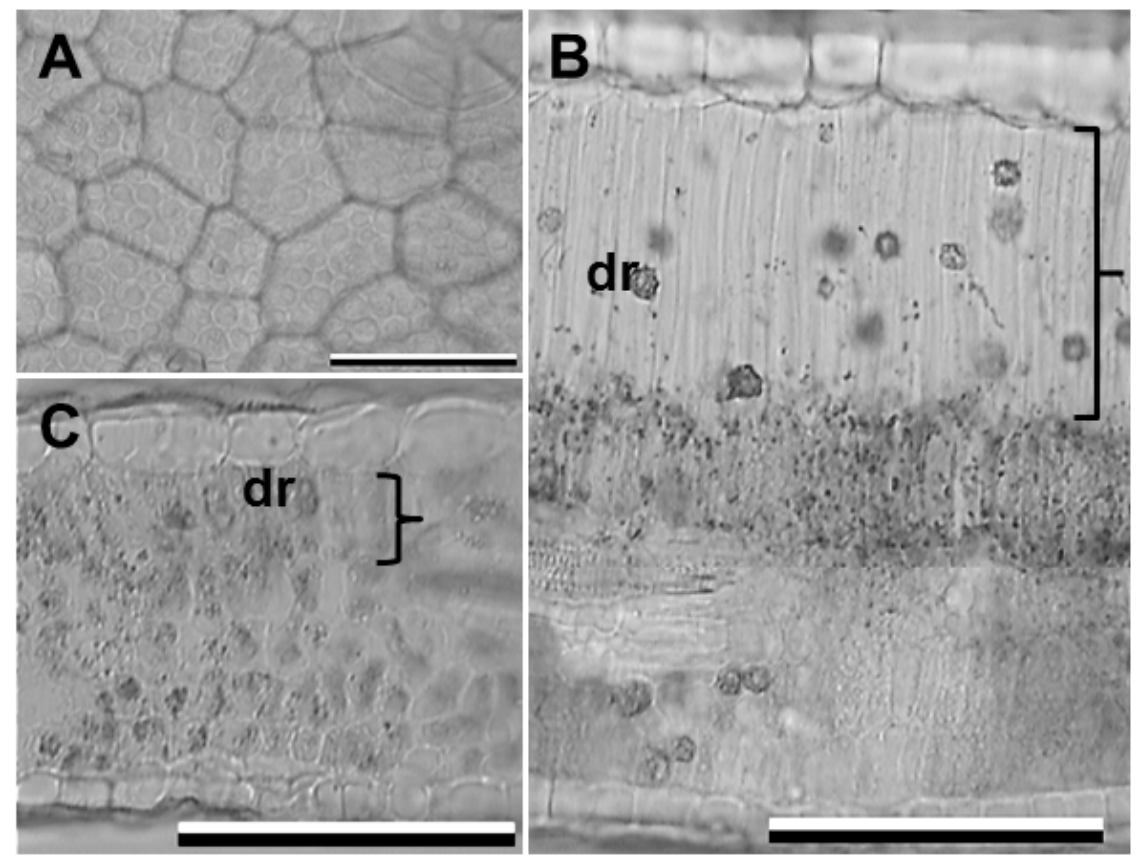

Fig. 4. Epidermis y mesófilo de Celtis ehrenbergiana en vista superficial y en corte transversal. A: Vista superficial de la epidermis y de células en empalizada subepidérmicas. B: Corte transversal en hoja de sol, se observa el mesófilo homogéneo en empalizada; con una llave se señaló el estrato adaxial de células en empalizada estrechas y largas. C: Corte transversal en hoja de sombra, se observa el mesófilo dorsiventral; con una llave se marcó el estrato adaxial de células en empalizada cortas. Abreviatura: dr=drusas. Escalas: A: $50 \mu \mathrm{m}$. B y C: $100 \mu \mathrm{m}$. 
vascular consta de un haz colateral rodeado por una vaina parenquimática. El tejido de sostén está conformado por colénquima adaxial y abaxial. Estas características generales de la vena media de la especie coinciden con la descripción de Dottori (1976). El colénquima adaxial y abaxial de la vena media en las hojas de sol está más desarrollado, es denso, con engrosamiento angular-masivo de sus paredes (Fig. 5A, C); mientras que, en las hojas de sombra el mismo está reducido, es laxo y de tipo angular-lagunar (Fig. 5B, D). La alta radiación solar produce un incremento del tejido de sostén que es un carácter xeromórfico como lo hallaron en diferentes especies (Roth, 1984; Ruíz \& al., 2007; Moreira \& al., 2012). Los cristales agrupados en forma de drusas se encuentran en el mesófilo foliar y en las venas en el parénquima que rodea los haces vasculares y en el floema como lo reportara Dottori (1976). Las drusas se encuentran en mayor número en las hojas de sol que en las de sombra (Fig. 4 B y C; Fig. 5A-D).
Fahn \& Cuttler (1992) refieren que es frecuente la presencia de cristales en hojas de ambiente xérico, por lo que el mayor contenido de drusas en las hojas de sol correspondería a un carácter xeromórfico desarrollado como adaptación a ambientes con alta radicación solar.

\section{Conclusiones}

En ambiente templado húmedo con baja radiación solar (hojas de sombra) las características foliares son mesomórficas: las células epidérmicas son proporcionalmente grandes y presentan paredes anticlinales ondulado-sinuosas, la lámina en la cara adaxial, no presenta o posee un número reducido de estomas ubicados sobre y en la proximidad de las venas; el mesófilo está compuesto por parénquima en empalizada adaxial y esponjoso denso abaxial; a nivel de la vena media el colénquima angular-lagunar

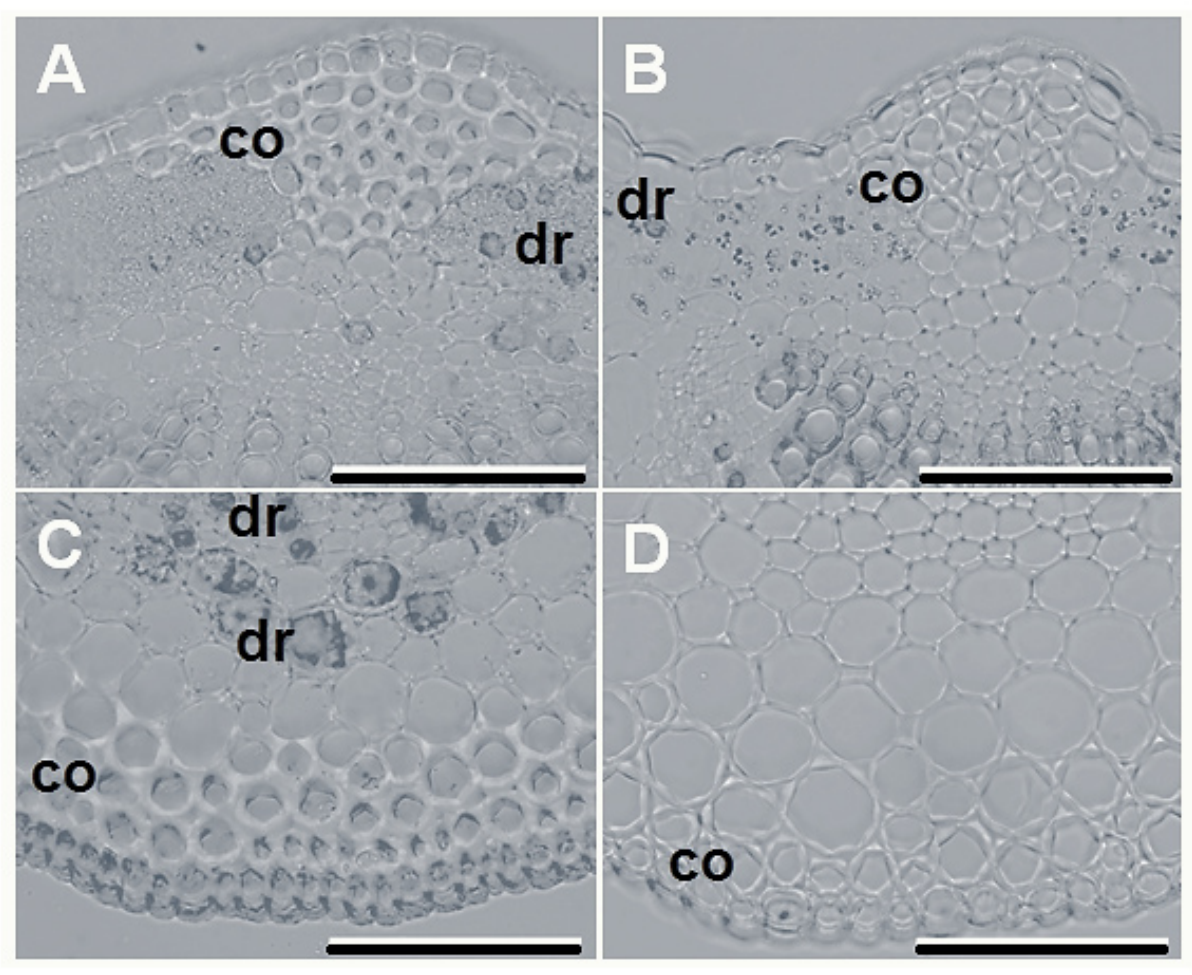

Fig. 5. Vena media de Celtis ehrenbergiana en corte transversal. A y C: Cara adaxial y abaxial, respectivamente de hojas de sol; en ambas, se observa colénquima angular-masivo, denso y drusas. B y D: Caras adaxial y abaxial, respectivamente de hojas de sombra; en ambas se observa colénquima angular-lagunar, laxo y drusas. Abreviaturas: co $=$ colénquima; $\mathrm{dr}=$ drusas. Escalas: $100 \mu \mathrm{m}$. 
es laxo. En ambiente templado húmedo con alta radiación solar (hojas de sol), las hojas desarrollan adaptaciones xeromórficas: las células epidérmicas alcanzan menor tamaño y sus paredes anticlinales son rectas a levemente curvadas, en especial en la cara adaxial; la lámina en la cara adaxial, presenta numerosos estomas ubicados en los espacios intercostales; el mesófilo está compuesto totalmente por parénquima en empalizada, las células en empalizada hacia la cara adaxial se alargan y el espesor de la lámina aumenta; a nivel de la vena media el colénquima angular sufre engrosamiento masivo de sus paredes y es denso. Los caracteres encontrados en la hoja de $C$. ehrenbergiana permiten establecer que las mejores condiciones para su crecimiento son las de un ambiente con alta radiación solar y disponibilidad de agua, donde sus hojas modifican la estructura y mejoran su eficiencia en la fotosíntesis. La variabilidad de los caracteres foliares hallados no solo indican una elevada plasticidad fenotípica de la especie, la cual le permite adaptarse morfo-fisiológicamente al ambiente en que vive, sino que además está en mejores condiciones que otras especies para adaptarse a los cambios climáticos.

\section{Agradecimientos}

Al personal del Departamento de Sismología e Información Meteorológica de la Facultad de Ciencias Astronómicas y Geofísicas, UNLP, por proveernos los registros climáticos.

\section{Bibliografía}

ABEDINI, W., P. BOERI, L. MARINUCCI, M. RUSCITTI \& L. SCELZO. 2000. Biotécnicas aplicadas a especies forestales nativas. Investigación Agraria: Sistemas y Recursos Forestales 9: 31-43.

ADEMA, M., S. SHARRY, S. BESTEIRO, P. GARCÍA TARTALO \& W. ABEDINI. 2007. Propagación in vitro de Celtis tala Gillies ex Planch. XXII Jornadas Forestales de Concordia, Entre Ríos. www.inta.gov. ar/concordia/info/Forestales/contenido/2007.htm (consultado: 10 de julio de 2010).

ARAMBARRI, A. M., M. C. NOVOA, N. D. BAYÓN, M.
P. HERNÁNDEZ, M. N. COLARES \& C. MONTI. 2011. Ecoanatomía foliar de árboles y arbustos de los Distritos Chaqueños Occidental y Serrano (Argentina). Bol. Soc. Argent. Bot. 46: 251-270.

ARTURI, M. F. \& J. F. GOYA. 2004. Estructura, dinámica y manejo de los talares del NE de Buenos Aires. En M. F. Arturi, J. L. Frangi \& J. F.Goya (Eds.), Ecología y Manejo de los bosques de Argentina. Edulp 10: $1-23$.

ATHOR, J. (ed.). 2009. Parque Costero del Sur-naturaleza, conservación y patrimonio cultural. Fundación de Historia Natural "Féliz de Azara". Buenos Aires. 528 pp.

BERG, C. C. \& S. V. DAHLBERG. 2001. A revision of Celtis subg. Mertensia (Ulmaceae). Brittonia 53: 66-81.

BIANCO, C. A., T. A. KRAUS \& A. C. VEGETTI. 2004. La hoja. Morfología externa y anatomía. Universidad Nacional de Río Cuarto, Universidad Nacional del Litoral, REUN. 196 pp.

BRAVO, S. J. \& A. GRAU. 1992. Variaciones de la densidad estomática en poblaciones de Alnus acuminata en un gradiente altitudinal. Lilloa 38: 39-45.

COLARES, M. N. \& A. M. ARAMBARRI. 2008. Ziziphus mistol (Rhamnaceae): morfo-anatomía y arquitectura foliar. Lat. Am. J. Pharm. 27: 568-577.

COSA, M. T. \& N. DOTTORI. 2010. Adaptaciones anatómicas de plantas medicinales a la diversidad de ambientes. X Simposio Argentina y XIII Simposio Latinoamericano de Farmacobotánica, Córdoba, Argentina. 73 pp.

D’AMBROGIO DE ARGÜESO, A. 1986. Manual de técnicas en histología vegetal. Hemisferio Sur, Buenos Aires. 86 pp.

DEMAIO P., U. O. KARLIN \& M. MEDINA. 2002. Árboles nativos del centro de Argentina, L.O.L.A. (Literature of Latin America), Buenos Aires. 210 pp.

DOTTORI, N. M. 1976. Morfología foliar en Celtis tala y $C$. pallida con especial referencia a los domacios. Kurtziana 9: 63-80.

ESAU, K. 1982. Anatomía de las plantas con semilla. Hemisferio Sur, Buenos Aires. 512 pp.

FAHN, A. \& D. F. CUTLER. 1992. Xerophytes. In Encyclop. Plt. Anat., Part 9, pp. 51-81. Gebrüder Borntraeger, Berlín.

FERNÁNDEZ HONAINE, M., A. F. ZUCOL \& M. L. OSTERRIETH. 2005. Biomineralizaciones de sílice en Celtis tala (Celtidaceae). Bol. Soc. Argent. Bot. 40: 229-239.

GAY, A. P. \& R. G. HURD. 1975. The influence of light on stomatal density in the tomato. New Phytol. 75: 37-46.

GOYA, J. F., L. G. PLACI, M. F. ARTURI \& A. D. BROWN. 1992. Distribución y características estructurales de los talares de la reserve de Biósfera "Parque Costero del Sur". Rev. Fac. Agron. La Plata 68: 53-64. 
HERNÁNDEZ, M. P. \& A. M. ARAMBARRI. 2010. Stomatal distribution, stomatal density and daily leaf movement in Acacia aroma (Leguminosae). Bol. Soc. Argent. Bot. 45: 273-284.

JOHANSEN, D. A. 1940. Plant Microtechnique. New York: McGraw-Hill Book Co.

LUNA, M. L. \& E. R. DE LA SOTA. 2003. Estructura foliar de Jodina rhombifolia (Santalaceae) y sus variaciones en relación al área de distribución. Iheringia 58: 3-12.

MA, Q. W., C. S. LI, F. L. LI \& S. V. VICKULIN. 2004. Epidermal structures and stomatal parameters of Chinese endemic Glyptostrobus pensilis (Taxodiaceae). Bot. J. Linnean Soc. 146: 153-162.

MÉRIDA, E. \& J.ATHOR (eds.). 2006. Talares bonaerenses y su conservación. Fundación de Historia Natural "Felix de Azara". Buenos Aires. 259 pp.

METCALFE, C. R. \& L. CHALK. 1979. Anatomy of the Dicotyledons. Vol. 1. Clarendon Press, Oxford. 276 pp.

MOREIRA, N. S., L. B. S. NASCIMENTO, M. V. LEALCOSTA \& E. S. TAVARES. 2012. Comparative anatomy of leaves of Kalanchoe pinnata and $K$. crenata in sun and shade conditions, as a support for their identification. Rev. Bras. Farmacogn. 23: 929-936.

MURACE, M., M. L. LUNA \& G. KEIL. 2007. Determinación de la durabilidad natural de la madera del tala (Celtis tala Gill. ex Planch.) mediante ensayos normalizados de laboratorio. XXII Jornadas Forestales de Entre Ríos, Concordia. INTA. http://www.inta. gov.ar/concordia/info/Forestales/contenido/pdf/2007/ (consultado: 10 de julio de 2010).

PARKHURST, D. F. 1978. The adaptive significance of stomatal occurrence on one or both surfaces of leaves. J. Ecol. 66: 367-383.

POOLE, I., J. D. B. WEYERS, T. LAWSON \& J. A. RAVEN. 1996. Variations in stomatal density and index: implications for palaeoclimatic reconstructions. Plant Cell Environ. 19: 705-712.

ROTH, I. 1984. Stratification of tropical forests as seen in leaf structure. In Encyclop Plt. Anat., Gebrüder Borntraeger, Berlin. 522 pp.

. 1990. Leaf structure of a Venezuela cloud forest (in relation to the microclimate). In Encyclop. Plt. Anat., Gebrüder Borntraeger, Berlin. 242 pp.

ROYER, D. L. 2001. Stomatal density and stomatal index as indicators of paleoatmospheric $\mathrm{CO}_{2}$ concentration. Rev. Paleobot. Palyno. 114: 1-28.

RUÍZ, A. I., M. I. MERCADO \& G. I. PONESSA. 2007. Morfología y anatomía foliar de Jodina rhombifolia (Hook. y Arn.) Reissek (Santalaceae). Lilloa 44: 7583.

SALISBURY, E. 1927. On the causes and ecological significance of stomatal frequency with special refence to the woodland flora. Phil. Trans. R. Soc. Lond., Ser B, 216: 1-65.

SILVA, H. E. \& P. SILVA. 2001. Anatomía del tejido fotosintético de diez taxa de Opuntia establecidos en el secano árido mediterráneo de Chile. Rev. Chil. de Hist. Nat. 74: 341-351.

SMITH, W. K., T.C. VOGELMANN, E. H. DELUCIA, D. T. BELL \& K. A. SHEPHERD. 1997. Leaf form and photosynthesis. Do leaf structure and orientation interact to regulate internal light and carbon dioxide? BioScience 47: 785-793.

SPAVENTO, E. M., G. D. KEIL, M. A. MURACE, M. ARTURI \& G. ACCIARECI. 2006. Estudios tecnológicos de la madera de Celtis tala Gill. ex Planch., nativo de la provincia de Buenos Aires. Ciencia 1: 16-20.

STACE, C. A. 1965. Cuticular studies as an aid to plant taxonomy. Bull. Br. Mus. (Nat. Hist.) Bot. 4: 1-78.

TORAL, M., A. MANRÍQUEZ, R. NAVARROCERRILLO, D. TERSI \& P. NAULIN. 2010. Características de los estomas, densidad e índice estomático en secuoya (Sequoia sempervirens) y su variación en diferentes plantaciones de Chile. Bosque 31: 157-164.

WOODWARD, F. I. 1987. Stomatal numbers are sensitive to increases in $\mathrm{CO}_{2}$ from preindustrial levels. Nature 327: 617-618.

ZULOAGA, F. O., O. MORRONE \& M. J. BELGRANO (eds.). 2008. Catálogo de las plantas vasculares del Cono Sur (Argentina, Sur de Brasil, Chile, Paraguay y Uruguay). Monogr. Syst. Bot. Missouri Bot. Gard. 107: 983 pp. St. Louis. (http://www.darwin.edu.ar/ (Consultado: 3 de enero de 2013).

Original recibido el 3 de junio de 2013; aceptado el 10 de octubre de 2013. 